\title{
Improving GNSS CORS Design: The CORSnet-NSW Adjustable Antenna Mount (CAAM)
}

\author{
Russell Commins and Volker Janssen \\ Survey Infrastructure and Geodesy, NSW Land and Property Information, Bathurst, Australia
}

\begin{abstract}
Global Navigation Satellite System (GNSS) Continuously Operating Reference Station (CORS) networks are being built and expanded around the world, contributing to the definition and realisation of geodetic reference frames as well as providing reliable and accurate positioning infrastructure for a wide range of applications. Depending on the purpose of the GNSS station, CORS antenna monuments typically vary from concrete pillars anchored to bedrock to masts attached to buildings. An antenna mount is used to connect the GNSS antenna to the monument. In all cases it is desired that the CORS antenna is oriented to True North in order to gain maximum benefit from GNSS antenna modelling. Other requirements generally include the unambiguous definition of the survey mark and Antenna Reference Point (ARP), and a zero or minimal antenna height. This paper introduces the CORSnet-NSW Adjustable Antenna Mount (CAAM), developed by NSW Land and Property Information (LPI) for CORSnet-NSW, LPI's rapidly growing GNSS CORS network covering the state of New South Wales, Australia. The CAAM was purposely designed to be incorporated into (rather than simply attached to) antenna masts located on buildings or free-standing pole monuments but can also be used for pillar monuments. Being free of removable parts, it is adjustable in order to orient the antenna to True North without introducing an antenna height, thereby allowing clear definition and maximum traceability of the survey mark and the ARP. LPI encourages adoption of the CAAM for use in other GNSS CORS networks.
\end{abstract}

Keywords: GNSS, CORS, CORSnet-NSW, antenna mount.

\section{Introduction}

An increasing number of Global Navigation Satellite System (GNSS) Continuously Operating Reference Station (CORS) networks is being established and expanded around the world (e.g. Rizos and Satirapod,
2011). These networks do not only contribute to the definition and realisation of global, regional and national geodetic reference frames but also provide reliable and accurate positioning infrastructure for a wide range of applications and a diverse group of users. Benefits of CORS networks include rationalisation of infrastructure, consistent and reliable connectivity to the national datum, and the ability to provide a degree of legal traceability for satellite-based positioning.

CORSnet-NSW is a rapidly growing network of GNSS CORS providing fundamental positioning infrastructure for New South Wales (NSW), Australia that is accurate, reliable and easy to use (Janssen et al., 2011a, 2011b). The network also supports regional geodesy initiatives by contributing to the Asia-Pacific Reference Frame (APREF) (GA, 2013) as well as Geoscience Australia's free online processing service AUSPOS (GA, 2012), and assists climate change research by augmenting long-term tide gauge observations (Janssen et al., 2013). CORSnetNSW is built, owned and operated by Land and Property Information (LPI), a division of the NSW Department of Finance and Services. Currently consisting of about 120 permanent stations tracking multiple satellite constellations, efforts are underway to expand the network to over 150 stations by the end of 2013 .

Figure 1 illustrates the coverage of CORSnet-NSW, showing stations that are operational (indicated by small triangles) as well as some planned stations (indicated by small circles). A $50 \mathrm{~km}$ radius around active stations is shown to illustrate the maximum recommended coverage area for single-base Real Time Kinematic (RTK) operation, while Network RTK (NRTK) coverage is shown as a striped, pink polygon in areas that have sufficient station density to support this technique. Currently (February 2013), 54\% of the area of NSW (and $98 \%$ of the population) is covered by the singlebase RTK service, while NRTK is available to $35 \%$ of the area of NSW (and 94\% of the population).

In order to classify permanent GNSS stations according to their purpose, the concept of a tiered hierarchy of GNSS CORS was proposed by Rizos (2007) and has 
since been widely accepted across Australia (Burns and Sarib, 2010). Tier 1 stations contribute to international or global geodesy initiatives, Tier 2 stations provide primary national geodetic infrastructure for datum definition and scientific research, and Tier 3 stations are state or private GNSS networks, often established for real-time precise positioning services. Further tiers of GNSS CORS have also been proposed, e.g. a CORS operated for the purpose of controlled traffic farming and not using the national datum or a temporary CORS bolted to the side of a demountable construction site building may be considered Tier 4 or lower (LPI, 2012).

While Tier 1 and Tier 2 sites generally require state-ofthe-art geodetic GNSS antennas and solid pillar monuments firmly anchored to bedrock, Tier 3 sites are often mounted on buildings and may use smaller surveytype antennas. CORSnet-NSW falls into the Tier 3 category. However, several CORSnet-NSW stations have been built to Tier 2 specifications with extra support from state and federal funds, allowing their participation in the scientific, national AuScope network (AuScope, 2011).

GNSS antenna mounts are the devices used to connect the GNSS antenna to the survey monument. This paper introduces the CORSnet-NSW Adjustable Antenna Mount (CAAM), developed by LPI for its CORSnetNSW network. The CAAM was purposely designed to be incorporated into antenna masts located on buildings or free-standing pole monuments but can also be used for pillar monuments. Being free of removable parts, it is adjustable in order to orient the antenna to True North without introducing an antenna height. This allows clear definition and maximum traceability of the survey mark and the Antenna Reference Point (ARP).

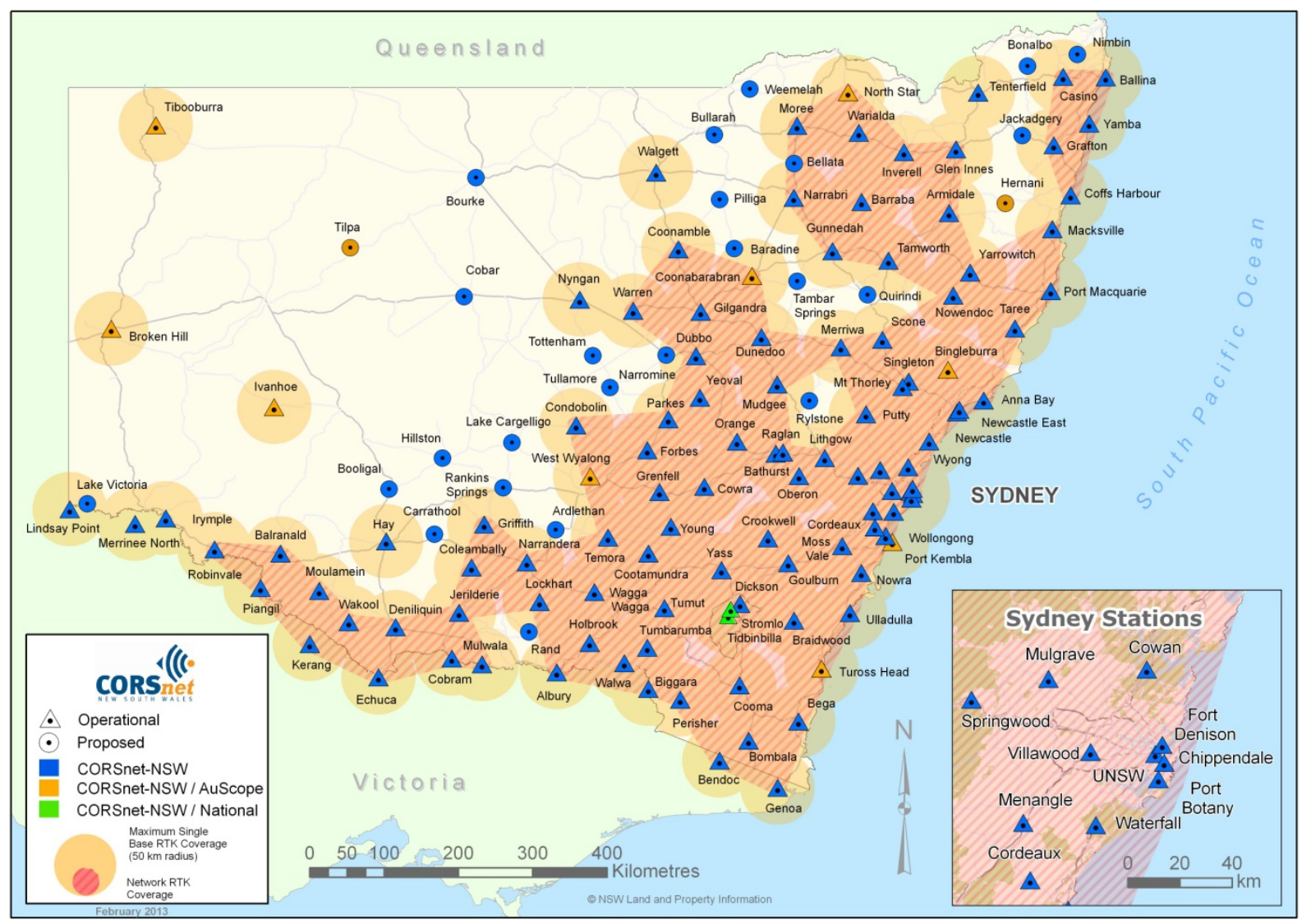

Figure 1: CORSnet-NSW network map as of February 2013 (LPI, 2013).

\section{GNSS Antenna Monuments}

The antenna monument secures the GNSS antenna mount to bedrock, the ground, a building or any other structure used as foundation. Tier 1 and 2 GNSS CORS antenna monuments should be structurally affixed to sound bedrock in order to provide data on tectonic plate motion, related tidal forces, inner-plate distortions (e.g. within Australia) and a precise realisation of the (inter)national geodetic reference frame. Reinforced concrete pillars and deep-drilled braced monuments are internationally recognised as the most stable and economic GNSS CORS structures acceptable for such sites (IGS, 2012). In Australia, the reinforced concrete pillar is the preferred monument for CORS network stations of national and international significance. 
Regional or state-wide CORS networks, such as CORSnet-NSW, generally utilise $3 \mathrm{~m}$ tall free-standing poles securely bolted to concrete slabs or concreted into the ground and 1-2 $\mathrm{m}$ tall wall monuments firmly attached to buildings (Figure 2). These monuments should have widths that are less than the antenna diameter to minimise multipath off the top surface of the monument and to ensure that antenna cables can be easily connected. The distance between the top of the antenna monument and the base of the GNSS antenna should ideally be zero. Generally, monument designs should not include or require any riser, spacer or adapter between the top plate and the antenna. Where unavoidable, their use should result in the antenna being less than $50 \mathrm{~mm}$ or greater than one GNSS carrier phase wavelength above the monument top plate (NGS 2013). This will reduce the influence of reflected GNSS signals (i.e. multipath effects). For some GNSS antennas, the monument top must either have a diameter of less than $85 \mathrm{~mm}$ or a bevelled edge to allow space for the antenna cable to pass.

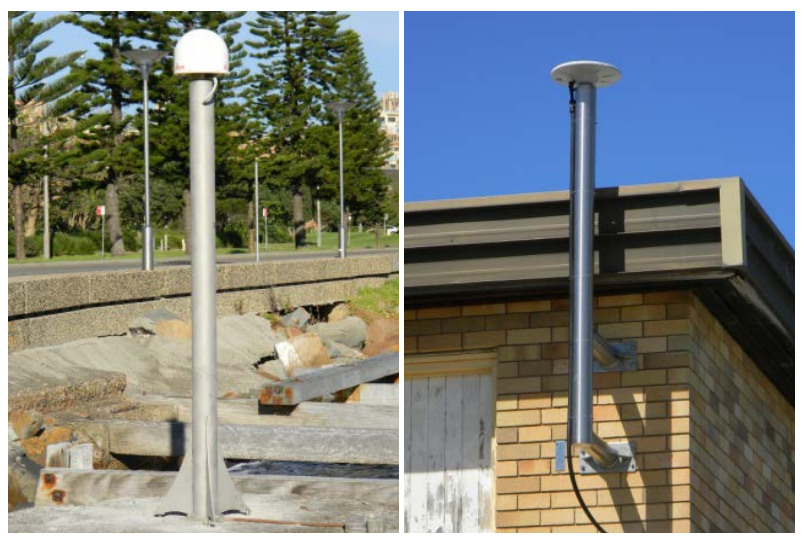

Figure 2: Free-standing pole and wall monuments at Tier 3 CORSnet-NSW sites.

For all GNSS CORS, the required characteristics of the monument generally include:

- Longevity and security.

- Appearance of being important infrastructure, i.e. not to be disturbed or tampered with.

- Sufficient elevation of the antenna to minimise obstructions occurring above the horizon.

- Stability in regards to short-term (e.g. vibration), medium-term (e.g. daily or seasonal movement) and long-term (e.g. subsidence and geophysical deformation) movements.

- Minimal thermal expansion.

- Corrosion and erosion resistance.

- Multipath minimisation.

- Simple design for ease of fabrication, installation and maintenance.

- $\quad$ True verticality (within $2 \mathrm{~mm}$ ) at the apex.
- Load-bearing capability (e.g. choke ring antennas typically weigh in excess of $4 \mathrm{~kg}$ ).

- Tamper-proof design.

- Low maintenance.

- Overall footprint and aesthetics (e.g. on historical buildings or at high-visibility locations).

- Light weight to minimise stress and damage to nonstructural parts of the building (building monuments only).

- Safety harness attachment points (tall monuments only).

\section{GNSS Antenna Mounts}

Antenna mounts connect the GNSS antenna to the monument. In all cases, it is desired that the CORS antenna is oriented to True North in order to gain maximum benefit from the latest GNSS antenna modelling. Other requirements generally include the unambiguous definition of the survey mark (supporting a clear definition of the ARP), a zero or minimal antenna height to remove or minimise potential complications and error sources, and the use of a truly vertical $5 / 8^{\text {th }}$ Whitworth thread spigot. It should be noted that the survey mark is generally defined as the intersection of the centre spigot and the horizontal top plate of the survey monument. Given the nature of metal and its tendency to warp when heat is applied through welding or galvanising, great care must be taken to ensure that a level mount top and vertical spigot are achieved.

In Australia, the antenna mount used with national (i.e. Tier 2) GNSS CORS pillar monuments is a stainless steel pillar plate concreted into place when the pillar top is built. This mount is oriented by placing the intended antenna on the mount and orienting the mount such that the antenna is correctly aligned to True North by employing a removable mounting bracket. The mount is then secured, the antenna removed, and the mount concreted in place (Figure 3). The four reference pins visible on the pillar top are later used to monitor the stability of the pillar at regular intervals (every 6-24 months) by high-precision terrestrial survey methods meeting specifications at the $<1 \mathrm{~mm}$ level (Janssen, 2009).

A number of other GNSS CORS antenna mounts exist, including the Southern California Integrated GPS Network (SCIGN) mount, the SECO 2072-series mount and the UNAVCO fixed-height mount (Figure 4). These mounts are inconvenient to use because they are required to be installed on top of the antenna monument and have the disadvantage of introducing an antenna height. In most cases, these mounts also contain removable parts, with negative implications on the traceability of the ARP. 

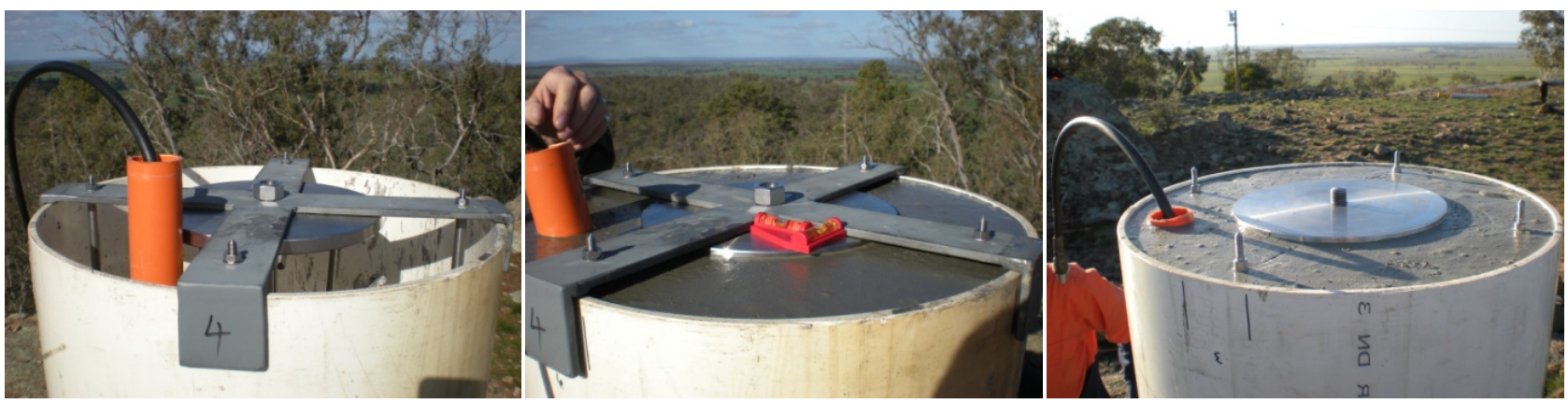

Figure 3: Construction of the GNSS CORS pillar plate at a Tier 2 CORSnet-NSW site.

(a)

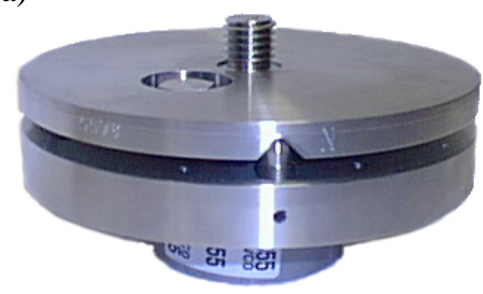

(b)

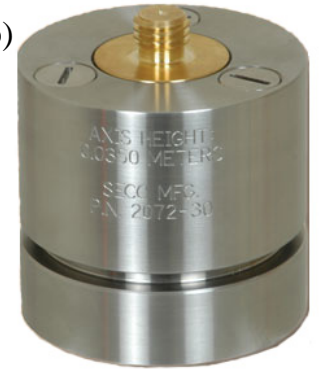

(c)

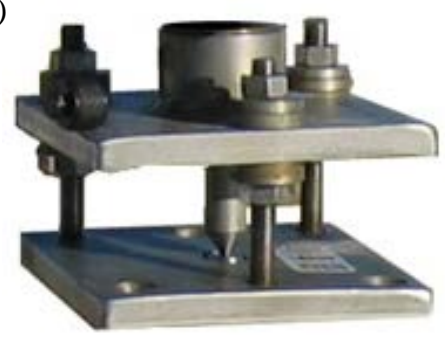

Figure 4: GNSS antenna mounts: (a) SCIGN mount, (b) SECO 2072 mount, (c) UNAVCO mount (UNAVCO, 2011).

\section{CAAM}

The CORSnet-NSW Adjustable Antenna Mount (CAAM) was developed by LPI and a patent has been issued (Australian Patent No. 2012200770). It was purposely designed to be incorporated into (rather than simply attached to) stainless steel antenna masts located on buildings and free-standing pole monuments during their manufacture, but can also be used for pillar monuments (LPI, 2012).

The CAAM (Figure 5) incorporates the following design specifications:

- Easily adjustable to True North without introducing an antenna height.

- Clear definition and maximum traceability of the survey mark.

- Integrated into the mount without any removable parts or entry points (e.g. to exclude insects).

- Simple to manufacture locally from readily available parts.

- Robust construction, made entirely from stainless steel and able to support heavy antennas.

- $\quad$ Adjustment procedure requiring minimal tools (i.e. one Allen key).

- Survey mark still usable if the adjustment mechanism should ever fail (worst case scenario).
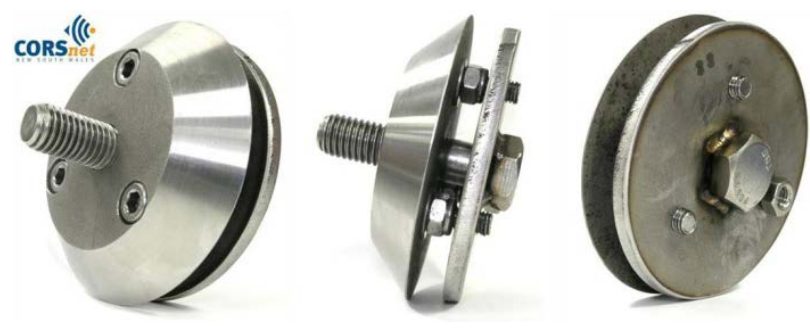

Figure 5: Internal workings of the CAAM.

Obviously, the GNSS antenna must be fully screwed onto the spigot in order to be stable. As such, the final orientation varies between different types of antenna and even between different individual antennas of the same type. By varying the amount of spigot protruding through the top plate slightly, the antenna can be fully screwed on and oriented to True North without the introduction of an antenna height (Figure 6).

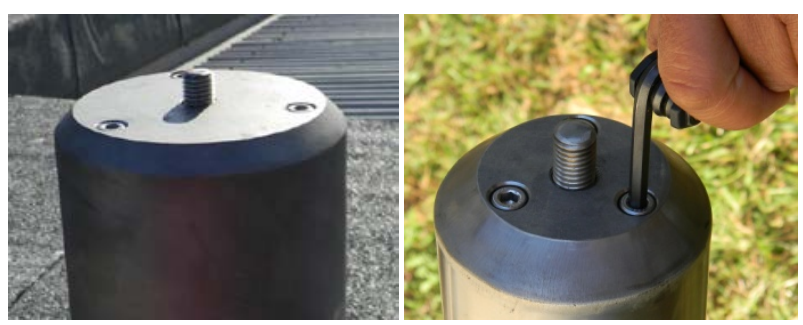

Figure 6: Adjustment of the CAAM, integrated into the GNSS antenna monument.

Turning the screws clockwise raises the centre spigot a tiny amount, allowing the antenna more rotation before it 
tightens. If one screw is rotated more or less than the others, the spigot will naturally try to tilt to one side. However, due to the thickness of the top plate it is not possible to adjust the three screws unevenly to any significant degree. A small tolerance allows the centre spigot enough freedom to move within the top of the mount during the adjustment process, while still keeping it vertical to within $0.25 \mathrm{~mm}$. Generally, a magnetic compass is used to orient the antenna to True North, remembering that the magnetic declination must be applied in this case.

The adjustment process of the CAAM spigot consists of the following steps:

1. The GNSS antenna is temporarily screwed on flush with the mount, noting how far the antenna is required to rotate (clockwise or anti-clockwise) before it faces True North.

2. The antenna is removed.

3. Each of the three adjustment screws is turned using an Allen key. If the antenna needs to rotate $30^{\circ}$ clockwise, each of the three screws is turned approximately $30^{\circ}$ clockwise.

4. The antenna is screwed on flush with the mount again and checked for direction.

5. This procedure is repeated until the antenna is firmly screwed on, faces True North, and the antenna

(a)
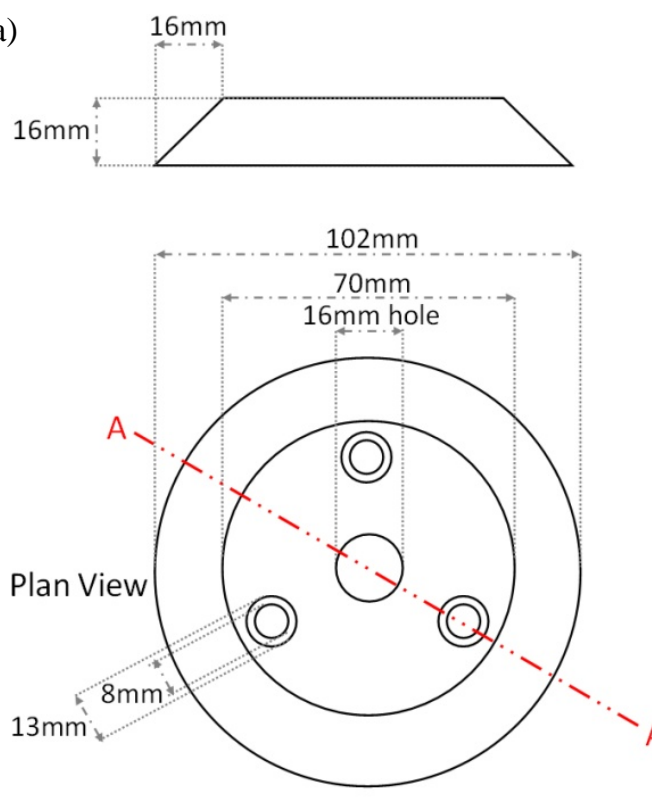

height is zero (i.e. no gaps between base of antenna and top of CAAM).

It is well understood that equipment changes at a GNSS CORS should be kept to a minimum in order to allow the collection of consistent data time series for scientific applications and to avoid changes in the antenna's position. However, the GNSS antenna will need to be replaced eventually, e.g. to accommodate new GNSS constellations and following hardware failure due to old age or a lightning strike.

Contrary to conventional antenna mounts, the CAAM allows the new antenna to be installed and oriented to True North without introducing (or changing) an antenna height. Consequently, the physical survey mark and the position of the ARP remain unchanged, allowing maximum traceability. Another feature of the CAAM is that the antenna's body covers the adjustment screws after it is installed. As such, the antenna prevents its orientation from being altered without being physically removed, which provides an additional level of security. Design sketches for the CAAM are freely available in LPI (2012) and reproduced in Figures 7 \& 8.

All new Tier 3 CORSnet-NSW stations installed by LPI since March 2011 include the CAAM, which has proven to be an excellent addition to the State's expanding CORS infrastructure. (b)

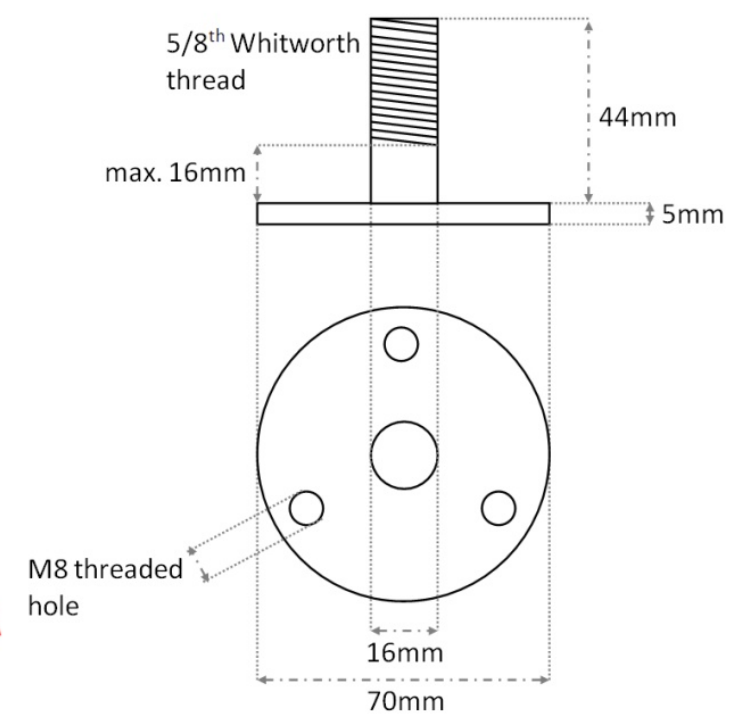

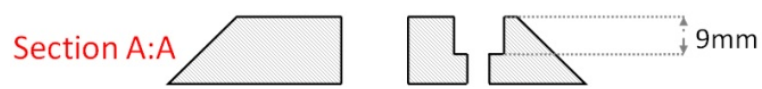

Figure 7: CAAM design sketches for (a) the top plate, and (b) the centre thread. 


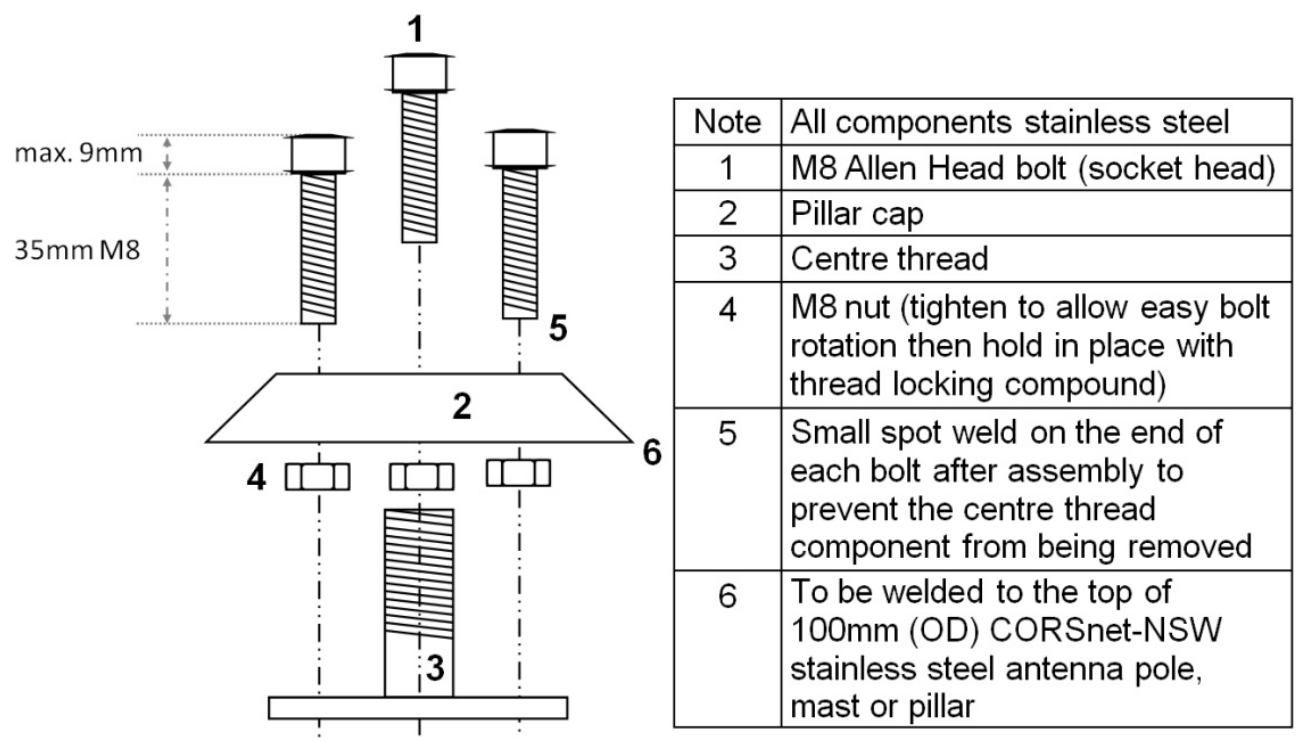

Figure 8: Design sketch for the assembly of the CAAM.

\section{Concluding Remarks}

Antenna mounts are an essential part of any GNSS CORS installation. Currently available GNSS antenna mounts are not ideal because they are required to be installed on top of the antenna monument, thereby introducing an antenna height. Often these mounts also contain removable parts, negatively affecting traceability of the survey mark and the ARP.

This paper has introduced the CORSnet-NSW Adjustable Antenna Mount (CAAM), which addresses these disadvantages by providing an easy-to-use alternative that is free of removable parts, integrated into the antenna monument, and adjustable to orient the antenna to True North. It was purposely designed for CORSnet-NSW installations using antenna masts attached to buildings and free-standing pole monuments. However, the CAAM can also be used for concrete pillar monuments, thereby eliminating the need to introduce an antenna height if an antenna is replaced. LPI encourages adoption of the CAAM for use in other GNSS CORS networks.

\section{References}

AuScope (2011) AuScope - An organisation for a national earth science infrastructure program, http://www.auscope.org.au/ (accessed Feb 2013).

Burns D. and Sarib R. (2010) Standards and practices for GNSS CORS infrastructure, networks, techniques and applications, Proceedings of XXIV FIG International Congress 2010, Sydney, Australia, April 11-16, 2010, 16 pp.
GA (2012) AUSPOS - Online GPS processing service, $\quad$ http://www.ga.gov.au/earthmonitoring/geodesy/auspos-online-gps-processingservice.html (accessed Feb 2013).

GA (2013) APREF, http://www.ga.gov.au/earthmonitoring/geodesy/asia-pacific-referenceframe.html (accessed Feb 2013).

IGS (2012) IGS proposed site guidelines, http://igs.org/network/guidelines/propose d.html (accessed Feb 2013).

Janssen V. (2009) Precision rules! How to establish an AusCORS site, Position, 44, pp. 64-66.

Janssen V., Commins R., Watson P. and McElroy S. (2013) Using GNSS CORS to augment long-term tide gauge observations in $N S W$, to appear in Proceedings of Surveying \& Spatial Sciences Institute Conference (SSSC2013), Canberra, Australia, April 15-19, 2013.

Janssen V., Haasdyk J., McElroy S. and Kinlyside D. (2011a) CORSnet-NSW: Improving positioning infrastructure for New South Wales, Proceedings of Surveying \& Spatial Sciences Institute Biennial Conference (SSSC2011), Wellington, New Zealand, November 21-25, 2011, pp. 395-409.

Janssen V., Haasdyk J., McElroy S. and Kinlyside D. (2011b) Technical challenges faced by CORS network operators: Experiences from New South Wales, Australia, International Journal of Geoinformatics, Vol. 7, No. 3, pp. 23-33. 
LPI (2012) Guidelines for CORSnet-NSW Continuously Operating Reference Stations (CORS), $\quad$ version $1.1, \quad$ available at http://www.lpi.nsw.gov.au/surveying/corsnet-

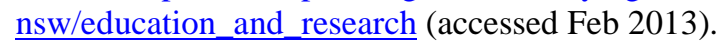

LPI (2013) CORSnet-NSW, http://www.corsnet.com.au/ (accessed Feb 2013).

NGS (2013) Guidelines for new and existing Continuously Operating Reference Stations (CORS), http://geodesy.noaa.gov/PUBS_LIB/CORS guidelines 2.0.pdf (accessed Feb 2013).

Rizos C. (2007) Alternatives to current GPS-RTK services \& some implications for CORS infrastructure and operations, GPS Solutions, Vol. 11, No. 3, pp. 151-158.

Rizos C. and Satirapod C. (2011) Contribution of GNSS CORS infrastructure to the mission of modern geodesy and status of GNSS CORS in Thailand, Engineering Journal, Vol. 11, No. 1, pp. 25-42.

UNAVCO (2011) UNAVCO resources: GNSS antenna mounts, http://facility.unavco.org/kb/questions/394/
UNAVCO+Resources\%3A+GNSS+Antenna+Mount s (accessed Feb 2013).

\section{Biographies}

Russell Commins (Russell.Commins@lpi.nsw.gov.au) is Project Officer (CORS) in the Survey Infrastructure and Geodesy branch at NSW Land and Property Information in Bathurst, Australia. He is a licensed communications technician, holds a Graduate Diploma (Applied Science) from Charles Sturt University, and has extensive experience in designing and building over 100 GNSS CORS.

Dr Volker Janssen (Volker.Janssen@lpi.nsw.gov.au) is Surveyor (Legal Metrology) in the same branch. He holds a Dipl.-Ing. (MSc) in Surveying from the University of Bonn, Germany, and a PhD in GPS for volcano deformation monitoring from the University of New South Wales (UNSW), Australia. Dr Janssen worked as a Lecturer in Surveying and Spatial Sciences at the University of Tasmania between 2004 and 2009. His research interests cover surveying and geodesy, with an emphasis on GNSS studies and CORS networks. 